\title{
Combined use of gliadins and SSRs to analyse the genetic variability of the Spanish collection of cultivated diploid wheat (Triticum monococcum L. ssp. monococcum)
}

\author{
Magdalena Ruiz $\cdot$ Edurne Aguiriano \\ Rosario Fité · Jose M. Carrillo
}

\begin{abstract}
This work studied the combined use of gliadins and SSRs to analyse inter- and intraaccession variability of the Spanish collection of cultivated einkorn (Triticum monococcum L. ssp. monococcum) maintained at the CRF-INIA. In general, gliadin loci presented higher discrimination power than SSRs, reflecting the high variability of the gliadins. The loci on chromosome $6 \mathrm{~A}$ were the most polymorphic with similar PIC values for both marker systems, showing that these markers are very useful for genetic variability studies in wheat. The gliadin results indicated that the Spanish einkorn collection possessed high genetic diversity, being the differentiation large between varieties and small within them. Some associations between gliadin alleles and geographical and agro-morphological data were found. Agro-morphological relations were also observed in the clusters of the SSRs dendrogram. A high concordance was found between
\end{abstract}

M. Ruiz $(\bowtie)$ - E. Aguiriano · R. Fité Instituto Nacional de Investigación y Tecnología Agraria y Alimentaria, Centro de Recursos Fitogenéticos, Autovía de Aragón $\mathrm{Km} \mathrm{36,} \mathrm{Apdo.}$ 1045,28800 Alcala de Henares, Madrid, Spain e-mail: mruiz@inia.es

J. M. Carrillo

Escuela Técnica Superior de Ingenieros Agrónomos, Dpto. Biotecnología, Ciudad Universitaria, 28040 Madrid, Spain gliadins and SSRs for genotype identification. In addition, both systems provide complementary information to resolve the different cases of intraaccession variability not detected at the agromorphological level, and to identify separately all the genotypes analysed. The combined use of both genetic markers is an excellent tool for genetic resource evaluation in addition to agromorphological evaluation.

Keywords Genetic variability - Germplasm . Gliadin alleles · Microsatellites - Triticum monococcum $\mathbf{L}$.

\section{Introduction}

Genetic variability of the Plant Genetic Resources of a country conditions breeder's activities and the development of cultivars with improved adaptation. In order to safeguard this genetic heritage, germplasm collections of many important crops are currently conserved ex situ in genebanks. In many cases, this is the only possible type of conservation because of social and economical impediments. However, ex situ collections may have a considerable percentage of duplicate accessions as well as entries misclassified (erroneous identification or accidental mixing) that difficult their effective conservation and use in breeding. The proper maintenance of 
genetic variation including heterogeneity of the accessions is one of the major aspects of genebank management. To resolve these problems it is essential to monitor intra- and inter-cultivar variability using polymorphic markers for genotype identification. This question is especially complex for cultivated einkorn wheat (Triticum monococcum L. ssp. monococcum), which has been considered a very uniform species (Kuspira et al. 1989). This diploid wheat, carrying the A genome, represents a potentially useful source of high protein content and tolerance to biotic and abiotic stresses for polyploid wheats (The 1973; Fedak 1985; Vallega 1992). Morphological and physiological characters, traditionally used, provide practical information to breeders but they cannot be sufficient because of low polymorphism and variation under environment. The electrophoretic spectra of gliadins, however, have proved to be highly polymorphic for genotype identification in einkorn (Metakovsky and Baboev 1992; Saponaro et al. 1995; Ciaffi et al. 1997; Alvarez et al. 2006). One difficult in using gliadin protein polymorphism is to reliably describe and compare complex gliadin spectra. Another disadvantage of these markers for distinguishing cultivars in T. monococcum $\mathrm{L}$. is that they are located at only two loci. Therefore, other polymorphic markers should be used to identify closely related cultivars. In recent years characterisation of germplasm by means of DNA fingerprinting techniques has supplied a tool for precise einkorn identification and a quantitative estimate of genetic diversity (Vierling and Nguyen 1992; Castagna et al. 1994; Le Corre and Bernard 1995). In this context, several authors have shown that microsatellites (SSRs) are genetic markers with uniform distribution in the wheat genome (Röder et al. 1998) and very useful for studying the variability of diploid wheat germplasm (Hammer et al. 2000; Medini et al. 2005). But, gliadins have in some aspects important advantages over DNA methods, in particular the possibility to monitor intra-varietal polymorphism by analysis of single grains and even half-grains. In addition, gliadin analysis is undoubtedly cheaper and less labour and time consuming. In spite of the fact that both types of markers could complement each other, at least in the field of wheat genotype identification, a direct comparison of the two methods on the same set of genotypes has not been performed.

The aim of this work is to analyse the combined use of gliadins and SSRs for the identification of cultivated einkorn genotypes, monitoring the intra-accession variability and to estimate the genetic diversity of the collection.

\section{Materials and methods}

\section{Materials}

The Spanish einkorn collection of Triticum monococcum L. ssp. monococcum maintained in the National Plant Genetic Resources Centre (CRF-INIA) were analysed in the present work. All the 17 landraces studied were released before 1960's and collected from 1931 until 1997. Passport data indicated that five accessions came from three provinces (Jaen, Cadiz and Huelva) in the South of Spain, other five came from the Centre of Spain (Cuenca province) and the rest (seven accessions) had unknown Spanish province origin. The accessions analysed identified by their bank number were: 1937, 1938, 13623, 13624, 13625 , $13626,13627,13628,13629,13630,14269,20465$, 20466, 20467, 20470, 29108, 29109.

\section{Gliadin analysis}

Gliadins were extracted from half single seeds and fractionated in acid ( $\mathrm{pH}$ 3.1) polyacrylamide gel electrophoresis (A-PAGE) according to Lafiandra and Kasarda (1985). As a minimum of eight grains per accession were analysed for intra-variety characterisation. Identification of the $G l i-A I^{m}$ and $G l i$ $A 2^{m}$ blocks in the protein spectra were performed following the work by Metakovsky and Baboev (1992). Only clear and contrast bands were considered. The durum wheat cultivar 'Langdon' was included as reference in all the gels.

Microsatellite (SSR) analyses

Plants germinated from the same grain analysed for gliadins were used to extract leaf DNA based on the method of Saghai-Maroof et al. (1984). 
Two accessions (BGE 13630 and BGE 20467) were not analysed because none half grain germinated. For the other 15 accessions 20 gliadin genotypes were examined for SSRs. Six primer pairs selected on the basis of their independent genomic distribution were used to amplify A genome (Table 4). Primers sequences, reaction mixture and PCR cycles were the same described by Röder et al. (1998). The forward primers of each primer pairs were fluorescently labelled with 6-FAM, HEX and TET fluorochrome tags. The allele size was analysed in an ABI PRISM 310 Genetic Analyser. The method described by Ghosh et al. (1997) was followed to determine the integer allele size.

\section{Agro-morphological traits}

The 17 accessions were sown for agro-morphological evaluation during 2001 in Alcala de Henares (Spain). One accession not germinated in the field (BGE 14269) but the rest were evaluated for the agro-morphological traits shown in Table 1 . The variables were measured according to IBPGR (1985). All the accessions were classified according to their botanical varieties (Dorofeev and Korovina 1979; Szabo and Hammer 1996).

\section{Statistical analysis}

To compare the gliadin and SSR polymorphism, the diversity indices calculated were: alleles per locus, effective number of alleles, Nei's diversity index and polymorphic information content (PIC). Gene diversity and PIC were calculated according to formulas of Nei (1973) and Anderson et al. (1993), respectively. To analyse gliadin gene diversity within and between the varieties total gene diversity over populations $(\mathrm{Ht})$ was divided into average gene diversity within (Hs) and between (Dst) populations (Nei 1973). The relative magnitude of gene differentiation between populations (Gst) was estimated as Dst/Ht.

Three dendrograms were made by UPGMA aggregation method with the distances of Rogers (1972) as modified by Wright (1978) for gliadin bands, Goldstein and Pollock (1997) for SSRs and Squared Euclidean for agro-morphological char-
Table 1 List of the agro-morphological traits used as descriptors

\begin{tabular}{|c|c|}
\hline Growth habit & Awnedness $(\mathrm{cm})$ \\
\hline 3 Prostrate & 1 Awless \\
\hline 5 Intermediate & 1.1; awnless (falling-out) \\
\hline 7 Erect & 2 Length: $1-3 \mathrm{~cm}$ \\
\hline Stem hairiness & 3 Length: $3-8 \mathrm{~cm}$ \\
\hline 1 In line & 4 Length: $>8 \mathrm{~cm}$ \\
\hline 2 All the stem & Spike density \\
\hline Top nude stem hairiness & $3 \mathrm{Lax}$ \\
\hline 1 Absent & 5 Intermediate \\
\hline 2 Low & 7 Dense \\
\hline 3 High & 9 Very dense \\
\hline Leaf hairiness & Glume hairiness \\
\hline 1 Hairless and hairy at & 1 Hairless \\
\hline the border & 1.1 Bright \\
\hline 2 Hairy in the surface & 1.2 Dull \\
\hline 3 Very hairy & 2 Low \\
\hline Spike length $(\mathrm{cm})$ & 3 High \\
\hline $\begin{array}{l}\text { Number of spikelets per } \\
\text { spike }\end{array}$ & $\begin{array}{l}\text { Glume colour } \\
1 \text { White }\end{array}$ \\
\hline Days to heading (days) & 2 Red to brown \\
\hline Days to maturity (days) & 3 Purple to black \\
\hline Plant height $(\mathrm{cm})$ & Seed colour \\
\hline Lemma awn barbs & 1 White \\
\hline 1 Rough & 2 Red \\
\hline 2 Smooth & 3 Purple \\
\hline \multicolumn{2}{|l|}{ Lemma awn colour } \\
\hline \multicolumn{2}{|l|}{1 White } \\
\hline \multicolumn{2}{|l|}{2 Black in the base } \\
\hline 3 Black & \\
\hline
\end{tabular}

acters. The distances for SSRs were calculated with the computer package SPAGeDI 1.1 (Hardy and Vekemans 2002) and the rest of the analyses with the NTSYS-pc software (Rohlf 1992). The correlation between the three distances matrices was tested with the Mantel $Z$ statistic (Mantel 1967). Significance of $Z$ was determined by comparing the observed $Z$ values with a critical $Z$ value obtained by calculating $Z$ for one matrix with out of 250 random permutations.

\section{Results}

Gliadin polymorphism

Twenty-two different electrophoretic patterns of gliadins were found in the 17 einkorn accessions analysed. Taking into account the work of Metakovsky and Baboev (1992), two independent gliadin blocks were determined for each accession. The upper block was composed by $\omega$ - and 
$\gamma$-gliadins, and in some cases also by one slowmoving $\beta$-gliadin, whereas the lower block was formed by $\beta$ - and $\alpha$-gliadins. These two blocks were encoded by the Gli-A1 $1^{m}$ and the Gli-A2m loci on the short arm of chromosomes 1 and 6, respectively. In total seven different blocks were identified at $G l i-A 1^{m}$ and 17 at $G l i-A 2^{m}$. The Fig. 1 shows some of these blocks. There was a high similarity in gliadin composition between the Gli-A1 ${ }^{m}$ blocks. Some of them differed only in one $\omega$ (e.g. $b$ and $d$, lines 2 and 3 ) or one $\beta$-gliadin (e.g. $d$ and $e$, lines 3 and 6 ). The most different $G l i-A 1^{m}$ blocks were the $G l i-A 1^{m} f$ and the $G l i-A 1^{m} g$ (lines 8 and 13). In contrast, Gli-A2 $2^{m}$ blocks shared less gliadin components. Table 2

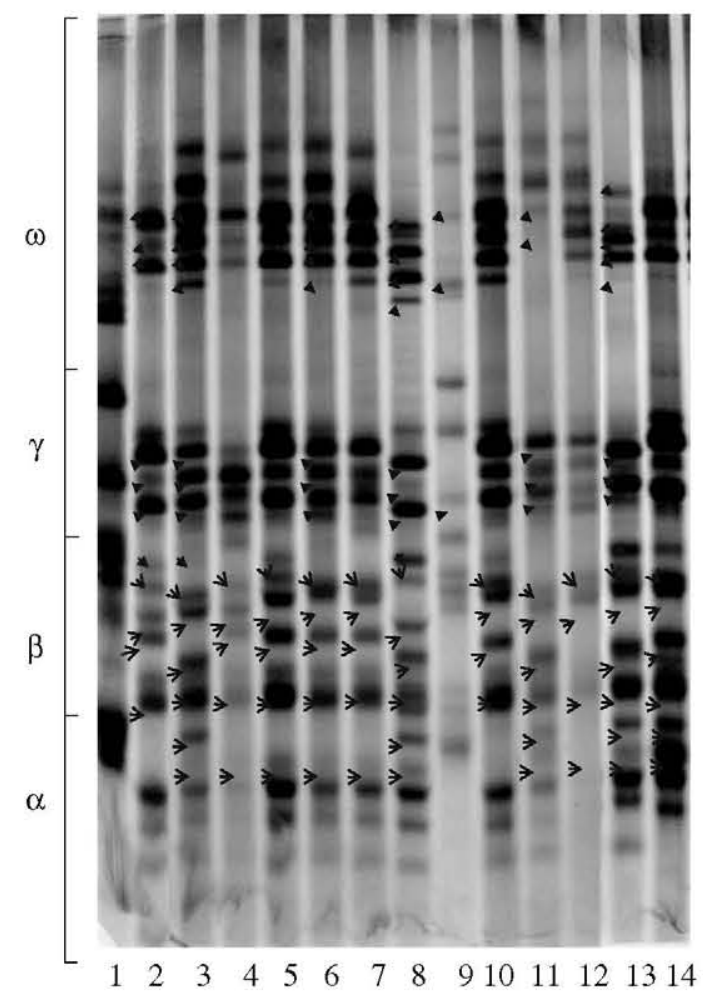

Fig. 1 Main gliadin genotypes of some Spanish accessions of T. monococcum L. ssp. monococcum. One durum and one bread wheat are included as test varieties. Arrowheads indicated $G l i-A 1$ alleles and arrows $G l i-A 2$ alleles. Lane 1, T. turgidum L. 'Langdon'; 2, Gli-A1 $1^{m} b$ Gli-A2 ${ }^{m} e ; 3$, Gli$A 1^{m} d$ Gli-A2 $2^{m} p ; 4$, Gli-A2 ${ }^{m} h ; 5$, Gli-A2 ${ }^{m} k ; 6$, Gli-A1 ${ }^{m} e$

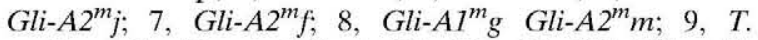
aestivum L. 'Candeal fino' Gli-A1o; 10, Gli-A2m g; 11, Gli-A1 $1^{m}$ a Gli-A2 $2^{m} p ; 12$, Gli-A2 $2^{m} i ; 13, G l i-A 1^{m} f$ Gli-A2 $2^{m} l$; 14, Gli-A2 $2^{m} n$ shows the allele frequencies in the 17 accessions analysed. The most frequent alleles were the Gli-A1 $1^{m} d(44 \%)$ and the $G l i-A 1^{m} e(26 \%)$, only different in one $\beta$-gliadin (Fig. 1, lines 3 and 6 ). The Fig. 2 shows the resemblance in component composition between the Gli-Alm $d$ block (line 4) and some Gli-A1 blocks of T. turgidum L. and T. aestivum L. For the Gli-A2 ${ }^{m}$, allele $a$ was the most frequent (Fig. 2, lane 5). The rest of the alleles were present in only one genotype and had low frequencies. Some $G l i-A 2^{m}$ alleles as $b, c, d$ and $o$ (Fig. 2, lane 4) were present only in biotypes.

Statistics of genetic diversity within and between varieties are given in Table 3 . The overall gene diversity in the entire sample was $\mathrm{Ht}=0.810$ with more than $90 \%$ of the diversity due to differentiation between varieties as opposed to within varieties. The two gliadin loci presented large genetic diversity, small genetic variation within varieties (Hs) and large interpopulation differentiation (Gst). The Gli-A2 $2^{m}$, the most polymorphic locus, displayed the largest gene diversity and intra-variety expected heterozygosity (Hs). This locus was more useful to distinguish between varieties (larger Dst) and it was less fixed within them (smaller Gst) than the Gli-A1 $1^{m}$.

All the main genotype (gt. 1) of the 17 accessions analysed were different but one duplication was found between the main genotype of one accession and the biotype of other one (20466 gt. 1 and 1937 gt. 4, Fig. 3).

\section{Microsatellites polymorphism}

Table 4 shows the list of the SSRs used, their map position, primer sequence according to Röder et al. (1998), number and effective number of alleles, and PIC values generated in the 15 einkorn accessions analysed. Table 2 shows the alleles amplified at the SSR loci and their frequency. From two to nine alleles per locus were identified with an average of 4.8 alleles (Table 4). The most polymorphic loci were the Xwms570 and Xwms136 on the long and short arm of the chromosomes $6 \mathrm{~A}$ and $1 \mathrm{~A}$, respectively. All the main genotypes identified with gliadins were distinguished with the SSR 
Table 2 Alleles and frequencies at the gliadin and SSR loci in the Spanish einkorn accessions at the CRF-INIA

\begin{tabular}{|c|c|c|c|c|c|c|c|c|}
\hline \multirow[t]{2}{*}{ Allele/locus } & \multicolumn{2}{|c|}{ Gliadin loci } & \multicolumn{6}{|l|}{ SSR loci } \\
\hline & $G l i-A 1^{m}$ & Gli-A2 $2^{m}$ & $X g w m 136$ & $X g w m 2$ & Xgwm601 & $X g w m 156$ & Xgwm570 & Xgwm332 \\
\hline$a$ & 0.058 & 0.183 & 0.050 & 0.050 & 0.700 & 0.950 & 0.050 & 0.650 \\
\hline$b$ & 0.044 & 0.022 & 0.050 & 0.150 & 0.300 & 0.050 & 0.200 & 0.350 \\
\hline$c$ & 0.066 & 0.014 & 0.050 & 0.550 & & & 0.050 & \\
\hline$d$ & 0.448 & 0.022 & 0.250 & 0.100 & & & 0.150 & \\
\hline$e$ & 0.264 & 0.029 & 0.400 & 0.100 & & & 0.200 & \\
\hline$f$ & 0.058 & 0.117 & 0.100 & 0.050 & & & 0.200 & \\
\hline$g$ & 0.058 & 0.058 & 0.050 & & & & 0.050 & \\
\hline$h$ & & 0.066 & 0.050 & & & & 0.050 & \\
\hline$i$ & & 0.029 & & & & & 0.050 & \\
\hline$j$ & & 0.058 & & & & & & \\
\hline$k$ & & 0.058 & & & & & & \\
\hline$l$ & & 0.058 & & & & & & \\
\hline$m$ & & 0.058 & & & & & & \\
\hline$n$ & & 0.058 & & & & & & \\
\hline$o$ & & 0.007 & & & & & & \\
\hline$p$ & & 0.117 & & & & & & \\
\hline$q$ & & 0.036 & & & & & & \\
\hline
\end{tabular}

markers. The only duplicate detected was for two genotypes different from the main genotypes (20470 gt. 2 and 29109 gt. 3, Fig. 4). In contrast, the two genotypes clustered together by their gliadin composition were separated with the SSRs analysis (20466 and 1937 gt. 4). The PIC varied from 0.09 to 0.84 with a mean of 0.53 (Table 4). No correlation was observed between the number of alleles and the motif and the repeat number of the units ( $r=0.638 \mathrm{~ns}$ ). However, the largest number of alleles was detected at the two loci with longer amplified fragment.

\section{Agro-morphological polymorphism}

Eleven accessions belonged to the var. macedonicum Papag., three to var. monococcum, one to var. vulgare Körn. and one to var. atriaristatum Flaksb. All accessions had hairless leafs, rough awn barbs, very dense spikes and red seeds. A great part had intermediate growth habit, stem hairs on line, bright hairless white glumes and white awns. Other characters as hairiness of the top nude stem, days to heading, plant height, awnedness, spike length and number of spikelets per spike showed higher variability. Figure 5 shows the dendrogram obtained with these data. It was observed that all the entries were different but a group of five cultivars belonging to var. macedonicum showed high similarity (G1 in Fig. 5). None intra-accession variability was detected.

Analysis of the heterogeneous accessions

Three accessions, 1937, 20470 and 29109, were heterogeneous. The accession 20470 contained two genotypes (gt. 1 and gt. 2) with a frequency of $5 / 8$ and $3 / 8$, respectively. Both had the same Gli-A1 ${ }^{m}$ allele but the main genotype had the $G l i-A 2^{m} q$ and the other the $G l i-A 2^{m} b$, differing mutually in four gliadins. Both genotypes showed shorter distances with other genotypes based on their dissimilarity in gliadin bands (Fig. 3). Based on microsatellite results the two genotypes differed in four loci. Also gt. 2 was identical to other genotype found in another heterogeneous accession (29109 gt. 3, Fig. 4). Considering the results of both markers, gt. 2 might be an off-type or admixture resulting from errors during the maintenance of the collection.

Accession 29109 possessed three genotypes, gt. 1,2 and 3 , with a frequency of $4 / 8,3 / 8$ and $1 / 8$, respectively. Gt. 2 and 3 differed from gt. 1 in both gliadin loci. These dissimilarities affected one band for the $G l i-A 1^{m}$ block, and two and one for gt. 2 and 3 , respectively, for the Gli-A2 $2^{m}$. The two last genotypes were different in one gliadin at 


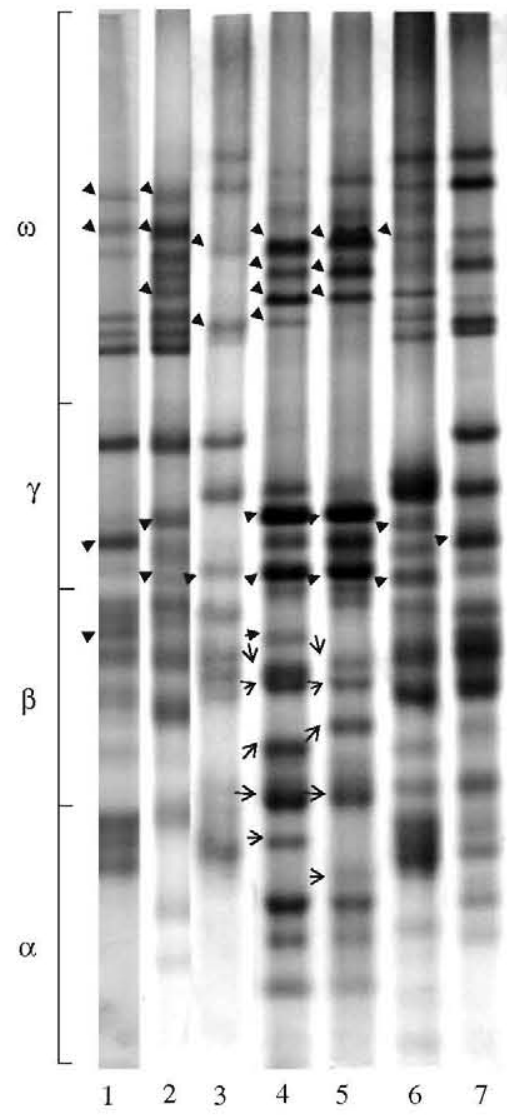

Fig. 2 A-PAGE of gliadins from different wheats. Arrowheads indicated $G l i-A 1$ alleles and arrows $G l i-A 2$ alleles. Lane 1, T. turgidum L. (Gli-AI $I^{d} c$, cv. 'Langdon'); 2, T. turgidum L. (Gli-A $I^{d} e$, cv. 'Kubanka'); 3, T. aestivum L. 'Candeal fino' (Gli-A1o); 4, Triticum monococcum L. ssp. monococcum (Gli-A1 $1^{m}$, Gli-A2 $\left.2^{m} o\right)$; 5, Triticum monococcum L. ssp. monococcum $\left(G l i-A 1^{m} c, G l i-A 2^{m} a\right) ; 6, T$. aestivum L. 'Candeal' (Gli-A1d); 7, T. aestivum L. 'Jeja' $($ Gli-Alp)

$G l i-A 2^{m}$. The three genotypes were grouped in the dendrogram (Fig. 3), but gt. 1 was closer to the accession 29108. Respect to the SSRs, gt. 1 differed in three and two alleles from gt. 2 and 3, respectively and gt. 2 and 3 differed mutually in two alleles. Gt. 3 was identical to 20470 gt. 2 and presented shorter distances with other genotypes than with gt. 2. Gt. 1 was also closely linked to the accession 29108 as in the gliadin analysis (Fig. 4). Based on these results it seems that 29108 and 29109 (gt. 1) are very closely related accessions, actually, they came from the same province but different locality. Gt. 2 and 3 might be a product of natural cross-pollination between accessions
Table 3 Gene diversity within and between einkorn varieties for each gliadin locus and for the overall 17 accessions

\begin{tabular}{lccllll}
\hline Locus & $\mathrm{A}^{\mathrm{a}}$ & $\mathrm{ne}^{\mathrm{b}}$ & $\mathrm{Ht}^{\mathrm{c}}$ & $\mathrm{Hs}^{\mathrm{d}}$ & $\mathrm{Dst}^{\mathrm{e}}$ & $\mathrm{Gst}^{\mathrm{f}}$ \\
\hline$G l i-A 1^{m}$ & 7 & 3.472 & 0.712 & 0.053 & 0.659 & 0.926 \\
$G l i-A 2^{m}$ & 17 & 10.996 & 0.909 & 0.101 & 0.808 & 0.889 \\
Mean & 12.0 & 7.2346 & 0.810 & 0.077 & 0.733 & 0.905 \\
\hline
\end{tabular}

${ }^{\text {a }}$ Alleles per locus

${ }^{\mathrm{b}}$ Effective number of alleles per locus

c Total gene diversity

d Average gene diversity within varieties

' Average gene diversity between varieties

${ }^{\mathrm{f}}$ Relative magnitude of gene differentiation between varieties

29108, 29109 and 20470. In fact, the three accessions were sown next to each other in the field.

Accession 1937 contained four genotypes with a frequency of $4 / 8,2 / 8,1 / 8$ and $1 / 8$ for gt.1,2, 3 and 4 , respectively. Gt. 1 and gt. 2 differed in three $G l i-A 2^{m}$ gliadins. Gt. 1 had one different $G l i-A 1^{m}$ gliadin, and five and three $G l i-A 2^{m}$ gliadins, respectively from gt. 3 and 4 . Gt. 2 was clustered together accessions 20470 and 1938 but gt. 1 was not grouped with any accession. Gt. 3 and 4 showed shorter distances with other entries (Fig. 3). SSRs data indicated that gt. 1 differed from gt. 2 and 4 in four and three loci, respectively (gt. 3 was not analysed). Similar to gliadin results, gt. 2 was closely related to accession 1938 , gt. 4 was grouped separately with other accessions while gt. 1 was very different from the rest of entries. These data indicated that gt. 2, 3 and 4 might be off-types.

Genetic relationships among different geographical regions

Some relations between gliadins and province origin of the landraces were observed. All the genotypes from the Jaen province (South of Spain) were grouped in Group 3 (G3, Fig. 3). All of them had the $G l i-A 2^{m}$ alleles $d, h$ (Fig. 1, lane 4) or $i$ (Fig 1, lane 12) no present in any other accession. These alleles had in common the presence of a $\beta$-gliadin (third $\beta$-gliadin of $G l i-A 2^{m}$ shown in lane 4 , Fig 1 ) or the absence of $\beta$-gliadins in this zone (Fig. 1, lane 12). Group 4 
Fig. 3 Dendrogram of 17 Spanish einkorn accessions calculated from gliadin data. Origin of the accessions: Centre (C) or South (S) of Spain is indicated.

Heterogeneous accessions are in bold

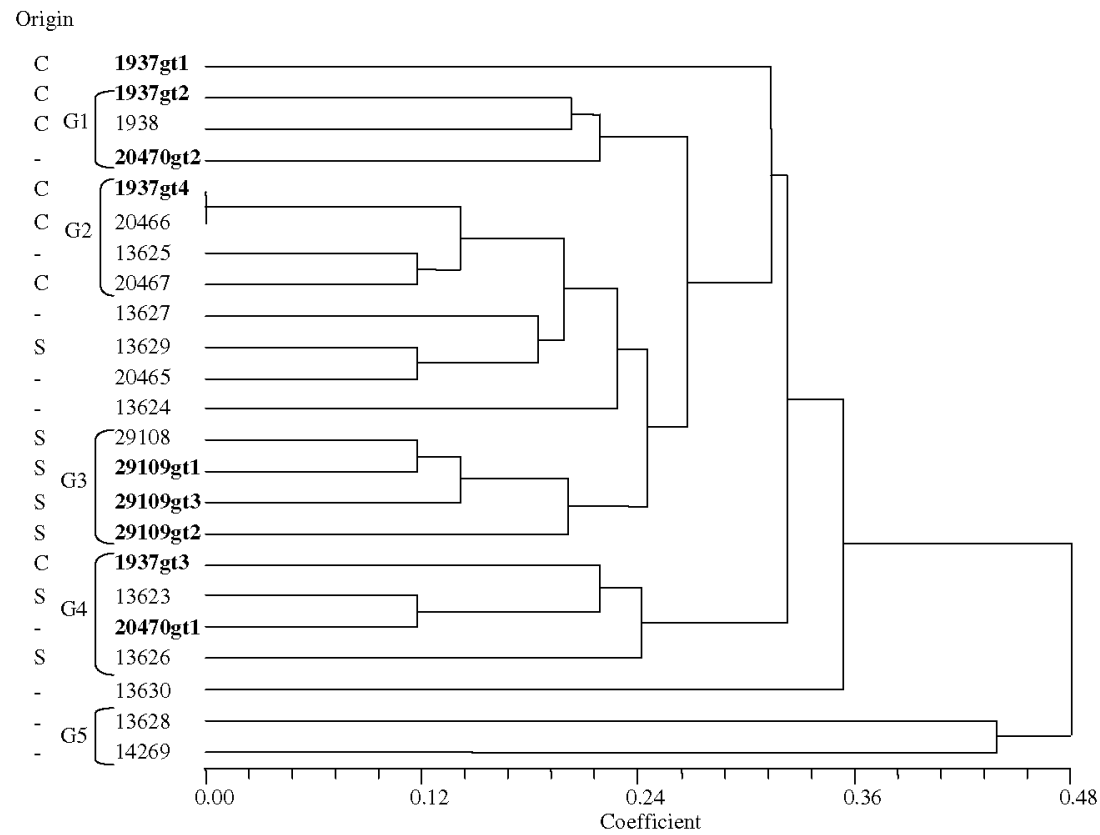

Table 4 SSR markers used, their map position, primer sequence, number and effective number of alleles and PIC values generated in the 15 einkorn accessions analysed

\begin{tabular}{|c|c|c|c|c|c|}
\hline $\begin{array}{l}\text { SSR } \\
\text { locus }\end{array}$ & $\begin{array}{l}\text { Chromosome } \\
\text { arm }\end{array}$ & Motif & $\mathrm{A}^{\mathbf{a}}$ & $n e^{b}$ & $\mathrm{PIC}^{\mathrm{c}}$ \\
\hline$X g w m 136$ & $1 \mathrm{AS}$ & $(\mathrm{CT}) 58$ & 8 & 4.081 & 0.755 \\
\hline$X g w m 2$ & $3 \mathrm{AS}$ & (CA) 18 & 6 & 2.857 & 0.650 \\
\hline Xgwm601 & $4 \mathrm{AL}$ & (CT) 17 & 2 & 1.724 & 0.420 \\
\hline$X g w m 156$ & $5 \mathrm{AL}$ & (GT)14 & 2 & 1.105 & 0.095 \\
\hline$X g w m 570$ & $6 \mathrm{AL}$ & (CT)14(GT) 18 & 9 & 6.451 & 0.845 \\
\hline$X g w m 332$ & $7 \mathrm{AL}$ & $(\mathrm{GA}) 36$ & 2 & 1.834 & 0.455 \\
\hline Mean & & & 4.8 & 3.009 & 0.536 \\
\hline
\end{tabular}

${ }^{\text {a }}$ Alleles per locus

${ }^{b}$ Effective number of alleles per locus

c Polymorphic information content

(G4) included the two accessions of the Cadiz province (South of Spain). These accessions were the unique with the $G l i-A 2^{m} p$ allele (Fig. 1, lanes 3 and 11). All the genotypes in G3 and G4 had in common a $\beta$-gliadin no present in other alleles. This band was the second $\beta$-gliadin of $G l i-A 2^{m}$ (lanes 3, 4, 11 and 12 of Fig. 1). Only 29109 gt. 2 did not have this gliadin, supporting that this genotype was not a biotype of the accession. Genotypes in Group 2 (G2) had the Gli-A2 $2^{m} a$ and they were from the Centre of Spain, except the 13625 from unknown province. Two entries from unidentified province clustered separately from the rest of accessions (G5) based on their difference in the $G l i-A 1^{m}$ alleles (Fig 1, lanes 8 and 13). In contrast to gliadin results, we have not observed geographical relations in the SSRs and agro-morphological dendrograms (Figs. 4 and 5).

\section{Relationships between characterisation data}

Mantel statistic $Z$ test was significant between gliadins and SSRs $(P=0.02)$ and no significant between agro-morphological data and gliadins $(P=0.21)$, and agro-morphological data and SSRs $(P=0.07)$. However, comparisons of the dendrograms showed some relationships between agro-morphological and gliadin data (Figs. 3 and 5 ). All the landraces analysed had a value of 230 or 234 for days to maturity. The six accessions of the earlier group came from the South of Spain, or had unknown origin, and all of them, except one, possessed the $G l i-A 1^{m} e$ allele in the main genotype. In contrast, most of the accessions of the later group possessed the $G l i-A 1^{m} d$ allele and came from the Centre of Spain or the province origin was unknown. Similar relationship was observed between number of spikelets per spike and gliadins. So, most of the accessions with higher values $(\geq 33)$ possessed the $G l i-A 1^{m} e$, the $\beta$ 
Fig. 4 Dendrogram of 15 Spanish einkorn accessions calculated from SSR data. Origin of the accessions: Centre (C) or South (S) of Spain is indicated. Heterogeneous accessions are in bold
Fig. 5 Dendrogram of 16 Spanish einkorn accessions calculated from agro-morphological data. Origin of the accessions: Centre (C) or South (S) of Spain is indicated
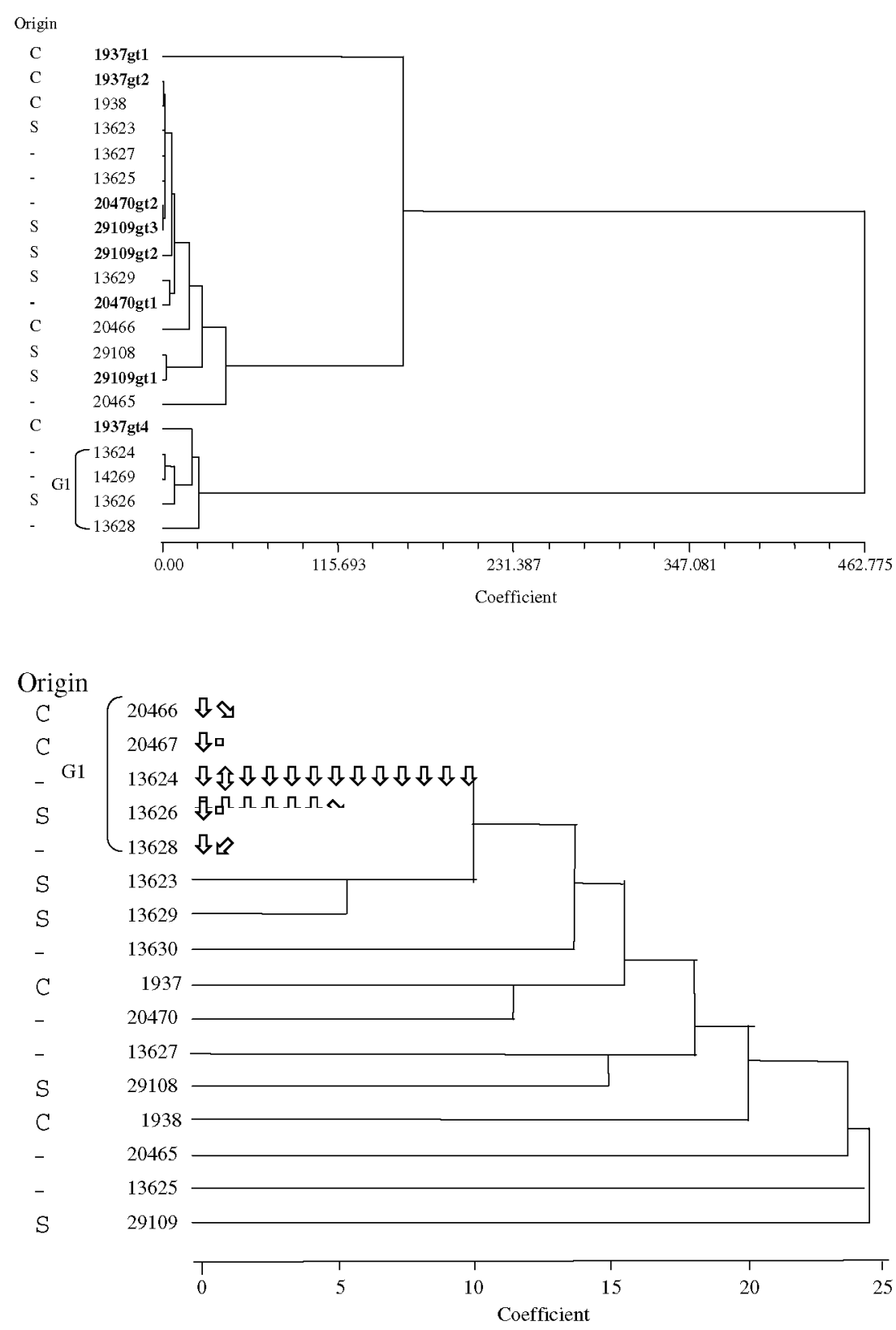

encoded at $G l i-A l^{m}$ was absent and came from the South of Spain. In contrast, the landraces with less spikelets $(<33)$ had the $G l i-A I^{m} d$ and the Gli$A 1^{m} \beta$-gliadin. These two associations were verified for the gt. 1 of the heterogeneous accession 29109 (earlier, $\geq 33$ spikelets per spike and Gli$A 1^{m} e$ ), but the gt. 2 and gt. 3 possessed the Gli$A 1^{m} d$ consistent with these genotypes would not be biotypes of the accession.
Based on SSR markers the accessions were closer related than with gliadins (Fig. 4). Only the group G1 was clearly separated based on their allele composition for the locus Xwms570. Some of these landraces were also grouped at the agromorphological level (G1, Fig. 5). All of them belonged to var. macedonicum and possessed intermediate growth habit, stem hairs on line and white awns shorter than $8 \mathrm{~cm}$. Two of these 
accessions were also separated by gliadins in G5 (Fig. 3) because of possessing rare alleles at Gli$A 1^{m}$. Also the main genotype of the accession 1937 was the most different with gliadins and SSRs.

\section{Discussion}

Metakovsky and Baboev (1992) determined that nearly all gliadin bands in einkorn wheat were inherited as two independent blocks. Since Gli$A 1$ and $G l i-A 2$ gliadin loci of polyploid wheats control the synthesis of blocks located in the same region of the electrophoretic spectrum (Metakovsky et al. 1984) it is assumed that the same loci are found in the diploid wheat Triticum monococcum L. Also, Metakovsky and Baboev (1992) found resemblance in component composition between the blocks of Triticum monococcum $\mathrm{L}$. ssp. monococcum and some blocks controlled by the A genome in polyploid wheats. Similar to these authors, we confirmed that blocks encoded at $G l i-A 1 d, o$ and $p$ in bread wheat, and present in Spanish landraces (Ruiz et al. 2002), shared some $\omega$ - and $\gamma$-gliadin with $G l i-A 7^{m}$ blocks in Spanish einkorn (Fig. 2). In the present work, a total of seven and 17 gliadin alleles were found at Gli$A 1^{m}$ and $G l i-A 2^{m}$, respectively in the 17 accessions analysed. These figures are comparable with those found by Saponaro et al. (1995) in 58 varieties (30 and 45 alleles), Metakovsky and Baboev (1992) in 109 entries (50 and 70 alleles) and Alvarez et al. (2006) in 22 Spanish einkorn accessions (7 and 14). Although comparisons between different gliadin spectra are not easy, some allele equivalences with this last work could be established since some accessions were shared. So, the $G l i-A 1^{m}$ alleles $a, c$ and $d$ corresponds to $f$, $e$ and $a$, respectively, and $G l i-A 2^{m} a, l, n$ and $p$ to $j, d, a$ and $f$, respectively, of Alvarez et al. (2006). However, some discrepancies were found in the mobility and components of these blocks. Some differences could be due to variations in the electrophoretic methods used. Another divergence was that we assumed that the slow $\beta$-gliadin present in some cultivars was encoded at $G l i-A 1^{m}$ (Fig. 1, lines 2, 3, 8 and 13) while Alvarez et al. (2006) located this gliadin at $G l i-A 2^{m}$. This gliadin presented a similar mobility to the $\beta$ gliadin coded at $G l i-A 1^{d} c$ in durum wheat (Fig. 1, line 1). Metakovsky and Baboev (1992) also showed that a slow $\beta$-gliadin was controlled by Gli-A1 ${ }^{m}$ in some einkorn accessions. The analysis of this gliadin together to the cv. 'Kubanka' (Fig. 2) indicated that this gliadin had a similar mobility than that found by Metakovsky and Baboev (1992). These results support that to compare and describe consistently gliadin patterns is the main difficulty in using gliadins. We have also found a resemblance in component composition of the $G l i-A I^{m}$ blocks except for the two accessions in Group 5 (lines 8 and 13, Fig. 3). $G l i-A 1^{m} \omega$ blocks had all of them two identical gliadins and the presence or absence of one to three additional bands. The most fast moving bands in $\alpha$ region were found in all the accessions analysed in agreement with Metakovsky and Baboev (1992). The Gli-A2 $2^{m}$ was the most polymorphic and the most useful to distinguish between varieties (Tables 3 and 4). Similar results were obtained in polyploid wheats (Kudryavtsev et al. 1996; Ruiz et al. 2002; Aguiriano et al. 2006) and in einkorn (Metakovsky and Baboev 1992; Castagna et al. 1994; Saponaro et al. 1995; Alvarez et al. 2006). In contrast, Ciaffi et al. (1997) found more allelic variation at $G l i-A 1^{m}$ than at $G l i-A 2^{m}$ (38 vs. 22) in 74 einkorn accessions.

A set of six microsatellite markers was used to characterise the different gliadin genotypes found. Alleles for each SSR locus were present in regular two or three base-pair steps, indicating that genetic variation during wheat evolution occurred in a continuous step-by-step manner. Gaps with one or more alleles were detected at two loci between the alleles with the lowest frequencies. The low number of accessions analysed could be the cause of these gaps. Three loci displayed the regular normal distribution of allele frequencies and the other three a bimodal distribution. According to Huang et al. (2002) it appears that natural selection plays a role in creating allelic variation at the loci with bimodal distributions. The alleles with higher frequencies might be selected and kept for adaptational reasons. Although the number of alleles was not correlated with the repeat number of the dimeric 
unit, the largest number of alleles was for the SSR with the largest repeat number and for the compound microsatellite with very long repeat units (Table 4). This last locus was also used by Medini et al. (2005), however, these authors failed in its amplification in einkorn. Since these authors only analysed one einkorn accession, it was possible that it had the null allele. No correlation between the number of alleles and the motifs of microsatellites was detected in agreement with Huang et al. (2002) in bread wheat. Similar to gliadin results the SSR locus mapped on chromosome 6A (Xgwm570) was also the most polymorphic, showing the usefulness of markers located on this chromosome for genetic variability studies in wheat.

Genetic analyses have indicated that the Spanish cultivated einkorn collection maintained at the CRF is largely structured genetically with about $90 \%$ of the total genetic diversity occurring between accessions and small differentiation within varieties (Table 3 ). The high gene diversity found for gliadin alleles (0.81, Table 3) was higher than that found by Alvarez et al. (2006) of 0.565 and it was in agreement with that found in Spanish bread and durum wheat of 0.81 and 0.68, respectively (Ruiz et al. 2002; Aguiriano et al. 2006). Comparisons between the $\mathrm{Ht}$ of gliadins and the PIC of SSRs (0.536) indicated that the discrimination power of gliadin loci was superior (Tables 3 and 4). However, the PIC of 0.78 for the two SSRs mapped on chromosomes $1 \mathrm{~A}$ and $6 \mathrm{~A}$ was similar to that obtained with gliadins. Le Corre and Bernard (1995) obtained a PIC of 0.234 for 20 RFLPs loci and 11 accessions from different countries supporting the high uniformity of this crop.

The two genetic marker techniques gliadins and SSRs were able to discriminate between all the main genotypes studied (gt. 1). Moreover, all the 20 genotypes were identified separately when the two approaches were used, indicating that both methods provided additional genetic information. Mantel test detected an association between both genetic markers. According to Bohn et al. (1999) these correlations can be found for related cultivars or if linkage disequilibrium exists between the different markers loci. In our

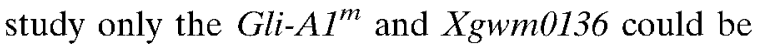

linked, so this correlation could be due to all the accessions are from Spain. In fact, Flaksberger (1935) classified T. monococcum L. in three ecomorphological groups and one of them was the Ibericum group (Spain, southern France and Morocco). Some associations were found between $G l i-A 2^{m}$ alleles and the origin of the accessions and $G l i-A I^{m}$ alleles and some agro-morphological traits. Some relations between Spanish origin (North or South of Spain) and gliadin alleles were also found in durum wheat landraces (Aguiriano et al. 2006). Gliadin genes, not subjected to direct selection by breeders, could be linked to genes directly selected under different environmental conditions. Similar to Hammer et al. (2000) some agro-morphological relations were observed in the clusters of the SSRs dendrogram.

Monitoring of intra-accession variability is an essential question for genebank experts. Gene flow between accessions due to outcrossing during regeneration or methodological errors during seed handling will cause unwanted contaminations. In this work, three heterogeneous accessions contained two, three or four genotypes identified with gliadins and with SSRs, and no detected at the agro-morphological level. The analysis together of gliadin and SSRs data indicated that they might be admixtures or result of natural cross-pollination between different accessions. Although a concordance between both markers was obtained the complementary information provided was very useful to resolve the different cases and to distinguish authentic biotypes from contaminations.

The combined use of gliadins and SSRs is an excellent tool for the identification of cultivated einkorn genotypes, mainly for verifying duplicates, and for monitoring the genetic variability present in germplasm collections. The knowledge obtained with both type of markers about the distribution of alleles, especially of rare alleles, is also important to the creation of core collections. Both techniques possess complementary advantages. Genetic variability estimation based on SSRs is more informative because they map a wider genome area, but gliadins have shown to possess higher discriminant power and stronger associations with geographical and agro-morphological data. The lower cost (economic and 
personnel) of this last technique and the semiautomated genotyping of SSRs are another important aspects to be considered in genetic resources evaluation.

Acknowledgements This work was supported by the grant RFP2004-018 from the INIA of Spain and the AGL 2003-6382 from Comision Interministerial de Ciencia y Tecnología (CICYT) of Spain. E. Aguiriano has received a pre-doctoral scholarship from the INIA.

\section{References}

Aguiriano E, Ruiz M, Fité R, Carrillo JM (2006) Analysis of genetic variability in a sample of the durum wheat Spanish collection based on gliadin markers. Genet Resour Crop Evol 53:1543-1552

Alvarez JB, Moral A, Martín LM (2006) Polymorphism and genetic diversity for the seed storage proteins in Spanish cultivated einkorn wheat (Triticum monococcum L. ssp. monococcum). Genet Resour Crop Evol 53:1061-1067

Anderson JA, Churchill GA, Antrique JE, Tanksley SD, Sorrels ME (1993) Optimising parental selection for genetic linkage maps. Genome 36:181-188

Bohn M, Utz HF, Melchinger AE (1999) Genetic similarities among winter wheat cultivars determined on the basis of RFLPs, AFLPs, and SSRs and their use for predicting progeny variance. Crop Sci 39:228-237

Castagna R, Maga G, Perenzin M, Heun M, Salamini F (1994) RFLP-based genetic relationship of Einkorn wheats. Theor Appl Genet 88:818-823

Ciaffi M, Dominici L, Lafiandra D (1997) Gliadin polymorphism in wild and cultivated einkorn wheats. Theor Appl Genet 94:68-74

Dorofeev VF, Korovina ON (eds) (1979) Wheat. Flora of Cultivated Plants (Russ., Engl. summary). 'Kolos', Leningrad

Flaksberger KA (1935) Wheat. Flora of Cultivated Plants (Russ) Vol I, Leningrad

Fedak G (1985) Alien species as sources of physiological traits for wheat improvement. Euphytica 34:673-680

Ghosh S, Karanjawala ZE, Hauser ER, Ally D, Knapp JI, Rayman JB, Musick A, Tannenbaum J, Te C, Shapiro S (1997) Methods for precise sizing, automated binning of alleles, and reduction of error rates in largescale genotyping using fluorescently labeled dinucleotide markers. Genome Res 7:165-178

Goldstein DB, Pollock DD (1997) Launching microsatellites: a review of mutation processes and method for phylogenetic inference. Genetics 139:463-471

Hammer K, Filatenko AA, Korzun V (2000) Microsatellite markers - a new tool for distinguishing diploid wheat species. Genet Resour Crop Evol 47:497-505

Hardy OJ, Vekemans X (2002) SPAGeDi: a versatile computer program to analyse spatial genetic structure at the individual or population levels. Mol Ecol Notes 2:618-620
Huang XQ, Börner A, Röder MS, Ganal MW (2002) Assessing genetic diversity of wheat (Titicum aestivum L.) germplasm using microsatellite markers. Theor Appl Genet 106:699-707

IBPGR (1985) Revised Descriptor list for wheat (Triticum ssp). International Board for Plant Genetic Resources, Rome, Italy

Kudryavtsev AM, Boggini G, Benedettelli S, Illichevskii NN (1996) Gliadin polymorphism and genetic diversity of modern Italian durum wheat. J Genet Breed 50:239-248

Kuspira J, Maclagan J, Bhambhani RN, Sadasivaiah RS, Kim NS (1989) Genetic and cytogenetic analyses of the A genome of T. monococcum L. V. Inheritance and linkage relationships of genes determining the expression of 12 qualitative characters. Genome 32:869-881

Lafiandra D, Kasarda DD (1985) One and two-dimensional (two-pH) polyacrylamide gel electrophoresis in a single gel: separation of wheat proteins. Cereal Chem 62:314-319

Le Corre V, Bernad M (1995) Assessment of the type and degree of restriction fragment length polymorphism (RFLP) in diploid species of the genus Triticum. Theor Appl Genet 90:1063-1067

Mantel N (1967) The detection of disease clustering and a generalized regression approach. Cancer Res 27:209220

Medini M, Hamza S, Rebai A, Baum M (2005) Analysis of gentic diversity in Tunisian durum wheat cultivars and related wild species by SSR and AFLP markers. Genet Resour Crop Evol 52:21-31

Metakovsky EV, Baboev SK (1992) Polymorphism and inheritance of gliadin polypeptides in T. monococcum L. Theor Appl Genet 84:971-978

Metakovsky EV, Novoselskaya AY, Kopus MM, Sobko TA, Sozinov AA (1984) Blocks of gliadin components in winter wheat detected by one-dimensional polyacrylamide gel electrophoresis. Theor Appl Genet 67:559-568

Nei M (1973) Analysis of gene diversity in subdivided populations. Proc Natl Acad Sci USA 70: 3321-3323

Röder MS, Korzun V, Wendehake K, Plaschke J, Tixier MH, Leroy P, Ganal MW (1998) A microsatellite map of wheat. Genetics 149:2007-2023

Rogers JS (1972) Measures of genetic similarity and genetic distance. In: Studies in Genetics VII. University of Texas, Austin, TX, pp 145-153

Rohlf FJ (1992) NTSYS-pe Numerical taxonomy and multivariate analysis system. Exeter software, NewYork

Ruiz M, Rodriguez-Quijano M, Metakovsky EV, Vazquez F, Carrillo JM (2002) Polymorphism, variation and genetic identity of Spanish common wheat germplasm based on gliadin alleles. Field Crops Res 79:185-196

Saghai-Maroof MA, Soliman K, Jorgensen RA, Allard RW (1984) Ribosomal DNA spacer-length polymorphism in barley: Mendelian inheritance, chromosomal location, and population dynamics. PNAS 81:80148018 
Saponaro C, Pogna NE, Castagna R, Pasquín M, Cacciatori P, Redaelli R (1995) Allelic variation at the Gli$A 1^{m}, G l i-A 2^{m}$ and $G l u-A 1^{m}$ loci and breadmaking quality in diploid wheat Triticum monococcum. Genet Res Camb 66:127-137

Szabó AT, Hammer K (1996) Notes on the taxonomy of farro: Triticum monococcum, $T$. dicoccon and $T$. spelta. In: Padulosi S, Hammer K, Heller J (eds) Hulled wheats. Proc. First Int. Workshop. Castelvecchio Pascoli, pp 2-40

The TT (1973) Chromosome location of genes conditioning stem rest resistance transferred from diploid to hexaploid wheat. Nature New Biol 241:256
Vallega V (1992) Agronomical performance and breeding value of selected strains of diploid wheat, Triticum monococcum L. Euphytica 61:13-23

Vierling RA, Nguyen HT (1992) Use of RAPD markers to determine the genetic diversity of diploid wheat genotypes. Theor Appl Genet 84:835-838

Wright S (1978) Evolution and the genetics of populations, vol 4. Variability within and among natural populations. University of Chicago Press, Chicago 